\title{
Hiperostosis cortical infantil (enfermedad de Caffey)
}

Delgado W, Arrascue M. Hiperostosis cortical infantil (enfermedad de Caffey). Rev Estomatol Herediana 2004;14(1-2) : 82 - 83.

\section{RESUMEN}

Se presenta un caso de hiperostosis cortical infantil o enfermedad de Caffey diagnosticado en una niña de 5 meses de edad. El cuadro se caracterizó por hinchazón asintomática de la hemicara izquierda debido a engrosamiento periostal del cuerpo y rama ascendente del maxilar inferior. Se revisa la literatura, el diagnóstico diferencial y el manejo de esta rara enfermedad ósea.

Palabras clave: HIPEROSTOSIS CORTICAL CONGÉNITA.

Infantile cortical hyperostosis (Caffey's disease)

ABSTRACT

A case of Infantile cortical hyperostosis or Caffey's disease diagnosed in a infant girl of 5 month is reported. The clinical picture was characterized by a non tender swelling of the left side of the face due to thickening of both vestibular and lingual periosteum of mandible. The differential diagnosis and the management of this rare oseous disease is discussed. Also the scarce literature available about the disease is reviewed.

Keywords: CONGENITAL CORTICAL HYPEROSTOSIS.

Wilson A. Delgado Azañero' Manuel Arrascue Dulanto ${ }^{1}$

'Docente del Departamento Académico Medicina, Cirugía y Patología Oral.

Facultad de Estomatología. Universidad Peruana Cayetano Heredia.

\section{Introducción}

La hiperostosis cortical infantil es conocida también como enfermedad de Caffey, síndrome de Caffey-Silverman, hiperostosis cortical infantil familiar o hiperostosis cortical infantil esporádica. Es una enfermedad ósea rara que se caracterizada por hiperostosis de la corteza del hueso afectado, usualmente la mandíbula, la clavícula o el cúbito (1).

Es un desorden autolimitante que afecta a infantes en los primeros meses de vida. La presentación clásica de esta enfermedad incluye la triada de irritabilidad, hinchazón y lesiones óseas (2). Aunque la etiología es desconocida, se han reportado formas familiares y esporádicas (3-5).

La enfermedad de Caffey se considera que es un proceso inflamatorio que al inicio produce inflamación del periostio y de los tejidos adyacentes. Cuando la inflamación pasa, el periostio queda engrosado y se forma hueso inmaduro subperiostal. No existen pruebas de laboratorio específicas para diagnosticar esta enfermedad. En algunos casos, la velocidad de eritrosedimentación y los niveles de fosfatasa alcalina pueden estar elevados y también puede existir anemia y leucocitosis (5).
El estudio radiográfico es el método de diagnóstico más importante. Las radiografías muestran engrosamiento de la cortical debido al depósito lamelar de hueso sobre el periostio.

Debido a que la enfermedad de Caffey es una enfermedad rara, que en muchos casos afecta la mandíbula, se ha considerado importante reportar el presente caso con la finalidad de llamar la atención acerca de su diagnóstico y manejo.

\section{Reporte de caso}

En diciembre del año 2002, fue traída por su madre al Servicio de Medicina Oral y Maxilofacial de la Facultad de Estomatología de la Universidad Peruana Cayetano Heredia, una niña de 5 meses de edad, por presentar asimetría facial de tres meses de evolución debido a hinchazón asintomática de la zona correspondiente a la mandíbula izquierda (Fig. 1).

La zona afectada no mostraba signos inflamatorios y su palpación no producía dolor. Aparte de la deformación señalada, la niña no mostraba ninguna otra alteración local o sistémica. El examen intraoral no reveló la existencia de dientes erupcionados y a la palpación se notó una deformación indolora del fondo de surco vestibular que se extendía desde la zona canina al cuerpo y rama ascendente de la mandíbula.

Una radiografía póstero anterior del cráneo mostró un engrosamiento de todo el cuerpo mandibular izquierdo con preservación de las corticales vestibulares y linguales (Fig. 2). No se indicó ningún examen de laboratorio.

El cuadro clínico y las radiografías establecieron el diagnóstico de enfermedad de Caffey. Se explicó a la madre en forma detallada las características de la enfermedad y se le indicó traer a la niña para controles periódicos cada tres meses.

El examen clínico y radiográfico de la niña realizada después de 20 meses, en agosto del año 2004, confirmó el diagnóstico inicial de enfermedad de Caffey. Al examen clínico la deformación facial había desaparecido (Fig.3), la radiografía póstero-anterior mostraba una mandíbula simétrica sin alteraciones de ningún tipo (Fig. 4). La madre refirió que durante los meses transcurridos, la niña no había manifestado ninguna molestia y que su desarrollo desde el punto de vista general era normal.

\section{Discusión}

La hiperostosis cortical infantil fue 


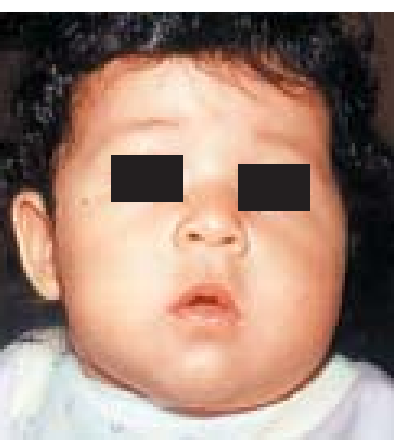

Fig. 1. Hinchazón asintomática de la zona mandíbular izquierda.

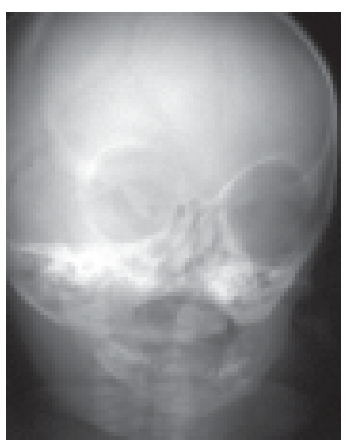

Fig. 2. Engrosamiento del periostio vestibular y lingual de la mandíbula.

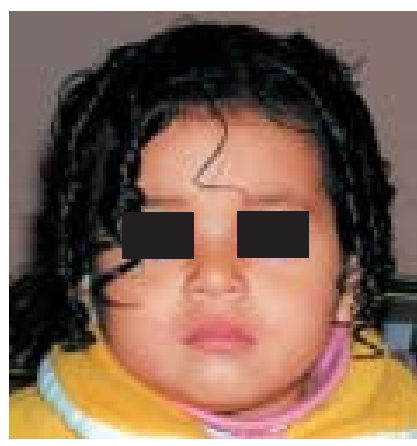

Fig. 3. Control clínico a los 18 meses: desaparición de la deformación facial.

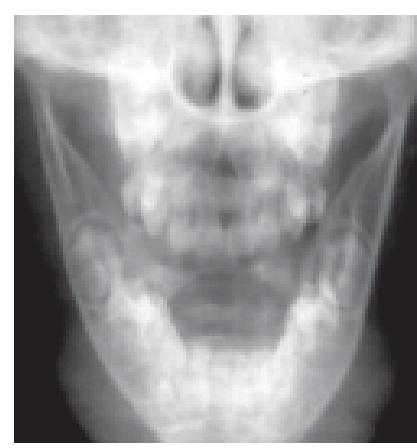

Fig. 4. Control radiográfico a los 18 meses: no hay alteraciones en el hueso mandibular izquierdo. descrita por Caffey en 1945. Es una enfermedad ósea rara que se caracterizada por hiperostosis de la corteza del hueso afectado. La mandíbula, la clavícula y el cúbito son los huesos más afectados, pero también se han reportado casos en otros huesos, tales como escápula, costillas, húmero, fémur, tibia, iliaco y metatarsos, además el compromiso óseo puede ser multifocal y asimétrico $(2,5)$.

Es un desorden autolimitante que afecta a infantes en los primeros meses de vida, generalmente aparece alrededor de los 5 meses de edad, pero también puede estar presente al momento de nacer. Es una entidad que produce cambios óseos, hinchazón de los tejidos blandos suprayacentes e irritabilidad (2). La etiología es desconocida, pero se reconoce la existencia de dos formas, familiar y esporádica (3-5).

La enfermedad de Caffey se considera que es un proceso inflamatorio que en los estadíos tempranos produce inflamación del periostio y de los tejidos adyacentes. Cuando la inflamación pasa, el periostio queda engrosado y se forma hueso inmaduro subperiostal. Los espacios medulares contienen tejido fibroso vascularizado. Posteriormente se observa hiperplasia lamelar del hueso cortical sin inflamación o cambios subperiostales

La enfermedad se inicia con hinchazón repentina, firme y profunda alrededor del hueso afectado que puede o no ser dolorosa. En algunos casos puede presentarse fiebre y cuando el compromiso es mandibular los niños rehusan comer.
No existen pruebas de laboratorio específicos para diagnosticar esta enfermedad, por lo que el diagnóstico está basado en las características clínicas y en el estudio radiográfico del hueso afectado.

El caso que reportamos corresponde a una niña de 5 meses de edad, cuya madre había notado desde hace tres meses hinchazón progresiva de la hemimandíbula izquierda y como la niña no manifestaba ninguna molestia no consulto inicialmente. Cuando es vista por nosotros comprobamos la presencia de una hinchazón profunda no dolorosa que comprometía la mitad del maxilar inferior izquierdo. El estudio radiográfico mostraba un engrosamiento más o menos simétrico en sentido vestibular y lingual que comprometía el cuerpo mandibular y rama ascendente a partir de la zona canina del lado izquierdo (Fig. 2).

El diagnóstico de enfermedad de Caffey se basó en la edad de la niña, la apariencia clínica y curso de la hinchazón y fundamentalmente en las características radiográficas, que mostraban engrosamiento de las corticales mandibulares debido al depósito lamelar de hueso sobre el periostio. Los resultados del control a los 20 meses confirmó el diagnóstico.

A pesar que el cuadro clínico y radiográfico es bastante característico debe considerarse en el diagnóstico diferencial cuadros de abuso infantil, traumatismo mandibular, osteomielitis del maxilar y también neoplasia que afectan a infantes como el sarcoma de Ewing y el neuroblastoma metastático.
Establecido el diagnóstico de enfermedad de Caffey en ningún caso se deben tomar biopsias. Cuando existe sintomatología dolorosa se puede indicar antiinflamatorios no esteroideos o corticoterapia teniendo en cuenta que esta medicación no tendrá ningún efecto sobre la lesión ósea. Lo más importante es controlar periódicamente al infante y explicar a los padres o tutores las característica autolimitantes de la enfermedad que termina con la resolución total del cuadro clínico en 6 a 12 meses sin dejar ninguna sequela.

\section{Referencias bibliográficas}

1. Van der Wall I. Diseases of the jaws: diagnosis and treatment. Denmark:Munksgaard, 1991:26-27.

2. Blank E. Recurrent Caffey's cortical hyperosostosis and persistent deformity. Pediatrics 1975; 55(6):85660.

3. Juniper RP. Caffey's disease. Br J Oral Maxillofac Surg 1982; 20:281-7.

4. Bernstein RM, Zaleske DJ. Familial aspects of Caffey's disease. Am J Orthop 1995; 24(10):777-81.

5. Saul RA, Lee WH, Stevenson RE. Caffey's disease revisited. Further evidence for autosomal dominant inheritance with penetrance. Am J Dis Child 1982;136(1):55-60.

\section{Dirección de Correspondencia}

Wilson A. Delgado Azañero

Honorio Delgado 430 Lima31-Perú

381-1950

wdelgado@upch.edu.pe 